\title{
Experimental Analysis of Ankle Foot Orthosis Using Pneumatic Artificial Muscle
}

\author{
Nurhanna Zulaikha Ishak ${ }^{1}$, Shahrol Mohamaddan ${ }^{1,2}$, Annisa Jamali ${ }^{1}$, Shinichiro \\ Yamamoto $^{2}$, Helmy Hazmi ${ }^{3}$, Low Cheng $\mathrm{Yee}^{4}$ and Akihiko Hanafusa ${ }^{2}$ \\ ${ }^{1}$ Faculty of Engineering, Universiti Malaysia Sarawak (UNIMAS), 94300 Kota Samarahan, \\ Sarawak, Malaysia \\ ${ }^{2}$ College of Systems Engineering and Science, Shibaura Institute of Technology, 3308570 \\ Saitama City, Japan \\ ${ }^{3}$ Faculty of Medicine and Health Sciences, Universiti Malaysia Sarawak (UNIMAS), 94300 \\ Kota Samarahan, Sarawak, Malaysia \\ ${ }^{4}$ Faculty of Mechanical and Manufacturing Engineering, Universiti Tun Hussein Onn \\ (UTHM), 86400 Parit Raja, Johor, Malaysia \\ mshahroleunimas.my
}

\begin{abstract}
Ankle Foot Orthosis (AFO) is a rehabilitation device which is used to assist patients with weakness over the ankle, foot and part of the leg especially when surgery is not warranted or during the recovery phase of the foot. In this research, the Pneumatic Artificial Muscle was used to develop and actuate the AFO to mimic the movement of the ankle, namely, plantarflexion, dorsiflexion and eversion. Gait pattern analysis was used to gauge the effectiveness of the AFO in human subjects. Two healthy subjects were recruited to simulate gait patterns. The gait analysis showed that the AFO actuated PAM was able to resist plantarflexion while assisting dorsiflexion. During the stance phase, the AFO were able to allow free ankle movement up to approximately $5^{\circ}$ dorsiflexion. It also allowed approximately $9^{\circ}$ eversion of the ankle from neutral position. Toe drag in the swing phase was avoided as the foot drop was prevented. In conclusion, the AFO performed well in providing support for a foot drop condition and this presents an opportunity for further testing in real patients with foot drop.
\end{abstract}

Keywords: Gait, Ankle Foot Orthosis, Dorsiflexion, Plantarflexion

\section{Introduction}

The foot is an important part of the human body that plays role in human mobility and weight bearing. Besides injury, the complexity of the foot causes difficulty in analyzing and understanding the mechanism of motion especially by means of quantification [1]. Neurological disorder such as trauma, stroke, spinal cord injury and cerebral palsy, may disrupt the function of the foot and thus the ability of human to walk properly. 